\title{
ON THE MICRO-TO-MACRO TRANSITION OF REINFORCEMENT SLIP IN TWO-SCALE MODELLING
}

\author{
ADAM SCIEGAJ, FREDRIK LARSSON, KARIN LUNDGREN AND KENNETH \\ RUNESSON \\ Chalmers University of Technology \\ Gothenburg, Sweden \\ e-mail: adam.sciegaj@chalmers.se \\ e-mail: fredrik.larsson@chalmers.se \\ e-mail: karin.lundgren@chalmers.se \\ e-mail: kenneth.runesson@chalmers.se
}

Key words: Bond, Slip, Multiscale, Lagrange Multipliers, Computational Homogenisation, Cracking

\begin{abstract}
A two-scale model for reinforced concrete, in which the macroscopic problem formulation is enriched by an effective reinforcement slip variable is considered. The corresponding subscale problem on the Representative Volume Element (RVE) is defined in terms of finding the response of the RVE subjected to effective variables (strain, slip, slip gradient) imposed from the macroscale. In this contribution, the two possible approaches of prescribing the effective reinforcement slip are discussed. Namely, a boundary definition of the macroscopic slip can be employed and the variable is thus prescribed only at boundary of the RVE, which corresponds to Dirichlet boundary conditions. Alternatively, a volumetric averaging measure can be used to define the effective reinforcement slip. In this case, the effective variables are imposed on the RVE in a weak sense via Lagrange multipliers. It is shown that the weak enforcement of reinforcement slip and its gradient resulted in objective interpretation of the effective variable (and its work conjugates), which was not pathologically dependent on the size of the RVE.
\end{abstract}

\section{INTRODUCTION}

Cracking of concrete at moderate load levels significantly influences the durability and service life of reinforced concrete structures, as cracks enable the ingress of harmful substances into the cross-section [1--3]. As a result, corrosion of reinforcement can occur. It is therefore vital to be able to model the cracking process in detail. In this aspect, not only cracking of the concrete must be captured by a suitable material model, but also the bond between steel and concrete plays an important role in the process. Thus, it needs to be taken into consideration in the analysis [4, 5].

Although steel-concrete interaction can be modelled in a number of ways, bond-slip models have been widely used for modelling the response of reinforced concrete structures. The bond-slip interaction can be modelled by direct resolution of the interface around rebars with interface elements. Although being straightforward (and readily available in most commercial codes), this approach requires much modelling effort, as the interface around every reinforcement bar must be resolved. For large structures, this can result in very large and computationally expensive models, which limits the method to small structures or parts of a structure. The computational cost can be reduced by employing multiscale modelling methods. In short, these methods enable the study of large- 
scale structures, full resolution models of which would be too expensive [6,7]. An example is the $\mathrm{FE}^{2}$ method [8,9], which connects the scales in a nested way, i.e. the macroscopic response is obtained via computational homogenisation performed on the subscale Representative Volume Elements (RVEs). Even though still computationally very expensive, this method is well suited for parallel computing, as all RVE problems can be solved independently of each other.

However, steel-concrete bond in conjunction with multiscale modelling has not been studied in the literature to the same extent [10, 11,13]. In recent works by the authors [12,13], a two-scale model of reinforced concrete, which considered the steel-concrete interaction was developed. Furthermore, it was enriched by a novel effective reinforcement slip macroscopic variable. This enrichment resulted in a localised effective strain field at the macroscale. In the model, the effective slip was prescribed only at the boundary of the RVE, which resulted in the pathologically RVE-size dependent effective response. Another possibility is to construct a volumetric definition of the effective reinforcement slip, whereby the macroscopic variable would be prescribed in the volume of the RVE in a weak (average) sense.

In this contribution, the two possible approaches of prescribing the effective reinforcement slip are further discussed. It is shown that the weak enforcement of reinforcement slip and its gradient results in more objective interpretation of the macroscopic variable (and its work conjugates), which is not pathologically dependent on the size of the RVE.

\section{TWO-SCALE MODEL}

In the $\mathrm{FE}^{2}$ method, the solution field is split into "smooth" (macroscopic) and "fluctuation" parts [14]. The problem can then be recast into a large-scale problem in terms of finding the response of the structure consisting of a homogeneous material with "effective" properties. This effective response is in turn obtained via computational homogenisation [15] of the response of a Representative Volume Element (RVE), which reflects the underlying heterogeneity and randomness of the material. The procedure is as follows: the large-scale (macroscopic) field is used as an input to the RVE problem. The boundary value problem on the RVE is then solved, and the subscale solution fields are homogenised (in practice, this is often synonymous to averaging) to produce the large-scale work conjugates. At the same time, the macroscopic tangent stiffness can be computed [16]. These effective values are then used to evaluate internal force vectors at the large-scale, and the procedure is repeated until the internal and external forces are balanced. Since in practice the numerical integration is performed at Gauss points, it is enough to consider the RVE at these locations only.

\section{LARGE-SCALE PROBLEM}

In the previous work of the authors, the large-scale problem on the effective displacement field, $\overline{\boldsymbol{u}}$ was enriched by an effective reinforcement slip variable, $\bar{s}$. This vectorial measure represented the translation of the reinforcement grid relative to the concrete. The effective quantities entering the subscale RVE problem were then identified to be the gradient of the displacement, i.e., the effective strain $(\bar{\varepsilon})$, as well as the effective slip $(\overline{\boldsymbol{s}})$ and its gradient $(\overline{\boldsymbol{g}})$. The governing partial differential equations for a structure subjected to traction $\hat{\boldsymbol{t}}$ at the external boundary $\Gamma_{\text {ext }}$ can be expressed as:

$$
\begin{aligned}
& \int_{\Omega} \overline{\boldsymbol{\sigma}}: \delta \overline{\boldsymbol{\varepsilon}} \mathrm{d} \Gamma=\int_{\Gamma_{\mathrm{ext}}} \hat{\boldsymbol{t}} \cdot \delta \overline{\boldsymbol{u}} \\
& \int_{\Omega} \overline{\boldsymbol{\tau}}_{\mathrm{b}} \cdot \delta \overline{\boldsymbol{s}}+\overline{\boldsymbol{\sigma}}_{\mathrm{s}}: \overline{\boldsymbol{g}} \mathrm{d} \Omega=0,
\end{aligned}
$$

where the effective work conjugates (effective stress $\overline{\boldsymbol{\sigma}}$, effective transfer stress $\overline{\boldsymbol{\tau}}_{\mathrm{b}}$, and effective reinforcement stress $\overline{\boldsymbol{\sigma}}_{\mathrm{s}}$ ) are obtained from computational homogenisation.

\section{SUBSCALE PROBLEM}

At the subscale, the material is modelled in detail, paying special attention to heterogeneity. In case of reinforced concrete, the reinforcement bars, concrete and the bond between 
them are considered. The structural problem on the RVE is posed in terms of finding the pertinent displacement fields in concrete $\left(\boldsymbol{u}_{\mathrm{c}}\right)$ and steel $\left(\boldsymbol{u}_{\mathrm{s}}\right)$. It is assumed, that the bond stress, $t_{\Gamma}$ is a function of reinforcement $\operatorname{slip}, \boldsymbol{e}_{1} \cdot\left(\boldsymbol{u}_{\mathrm{s}}-\boldsymbol{u}_{\mathrm{c}}\right)$, where $e_{1}$ is a unit vector in the direction along the reinforcement bar. Furthermore, the transverse reinforcement displacement is assumed identical to that of the surrounding concrete, i.e., $u_{\mathrm{s}, \perp}=\boldsymbol{e}_{\perp} \cdot \boldsymbol{u}_{\mathrm{s}}$.

The macroscopic quantities are imposed on the RVE via suitable boundary conditions. It is important that the boundary conditions fulfil so-called Hill-Mandel macrohomogeneity condition, i.e., that there is work equivalence across the scales. Two common types of boundary conditions fulfilling this criterion are the Dirichlet and Neumann type boundary conditions. For the Dirichlet boundary conditions, a macroscopic gradient can be imposed on the RVE directly by prescribing the value of the subscale field at the boundary of the RVE. In case of Neumann boundary conditions, no quantities are strongly prescribed for the RVE. Rather, we satisfy homogenisation conditions in a weak sense, e.g. by ensuring that the volume average of a subscale quantity is equal to its effective macroscopic value. 1 The way the micro-to-macro transition is defined is a modelling choice. Note that no such definition is needed in case of Dirichlet boundary conditions, as the macroscopic value comes directly from the boundary values. Here, we considered the two methods of applying the effective slip on the RVE.

\subsection{Boundary definition of slip}

In case of the boundary definition of slip (Dirichlet BC), the macroscopic variables $(\bar{\varepsilon}, \bar{s}$, $\overline{\boldsymbol{g}}$ ) are used to prescribe the deformation of both concrete and steel reinforcement at the bound- ary of the RVE, i.e.,

$$
\begin{aligned}
\boldsymbol{u}_{\mathrm{c}}= & \overline{\boldsymbol{\varepsilon}} \cdot[\boldsymbol{x}-\overline{\boldsymbol{x}}], \\
u_{\mathrm{s}, 1}= & \boldsymbol{e}_{1} \cdot \overline{\boldsymbol{\varepsilon}} \cdot[\boldsymbol{x}-\overline{\boldsymbol{x}}]+\boldsymbol{e}_{1} \cdot \overline{\boldsymbol{s}} \\
& +\boldsymbol{e}_{1} \cdot \overline{\boldsymbol{g}} \cdot[\boldsymbol{x}-\overline{\boldsymbol{x}}], \\
u_{\mathrm{s}, \perp}= & \boldsymbol{e}_{\perp} \cdot \overline{\boldsymbol{\varepsilon}} \cdot[\boldsymbol{x}-\overline{\boldsymbol{x}}],
\end{aligned}
$$

where $\boldsymbol{u}_{\mathrm{c}}$ is the deformation in the concrete, $u_{\mathrm{s}, 1}$ is the longitudinal displacement of steel reinforcement, and $u_{\perp}$ is the transverse displacement of reinforcement. The subscale problem can then be solved for the remaining degrees of freedom, and the effective work conjugates can be computed.

\subsection{Volumetric definition of slip}

In order to derive the formulation of the subscale problem, we must first define a suitable micro-to-macro ansatz. To this end, we consider the total displacement field in the form $\boldsymbol{u}=\left(\boldsymbol{u}_{\mathrm{c}}, \boldsymbol{u}_{\mathrm{s}}\right)$, and we define the functions $\overline{\boldsymbol{s}}_{\square}(\boldsymbol{u})$ and $\overline{\boldsymbol{g}}_{\square}(\boldsymbol{u})$. These modelling assumptions govern how the subscale variables are averaged to produce an effective macroscopic value. For the homogenisation condition of the slip, we simply take it to be the difference between the average longitudinal displacement in reinforcement and the average deformation of concrete in that direction. In the case of slip gradient we apply similar logic, the homogenised value being the the difference between average deformation gradients along steel and in concrete. To complete the formulation, we specify that the homogenised values shall be equal to macroscopic ones. These constraints are then applied on the equation system via Lagrange multipliers, i.e.,

$$
\begin{aligned}
-\overline{\boldsymbol{s}}_{\square}(\boldsymbol{u}) \cdot \delta \hat{\boldsymbol{\tau}} & =-\overline{\boldsymbol{s}} \cdot \delta \hat{\boldsymbol{\tau}}, \\
-\overline{\boldsymbol{g}}_{\square}(\boldsymbol{u}): \delta \hat{\boldsymbol{\sigma}}_{\mathrm{s}} & =-\overline{\boldsymbol{g}}: \delta \hat{\boldsymbol{\sigma}}_{\mathrm{s}},
\end{aligned}
$$

where the Lagrange multipliers $\hat{\tau}$ and $\hat{\sigma}_{\text {s }}$ can be shown to be equal to the effective work conjugates $\overline{\boldsymbol{\tau}}_{\mathrm{b}}$ and $\overline{\boldsymbol{\sigma}}_{\mathrm{s}}$, respectively. The equation

\footnotetext{
${ }^{1}$ Here, due to volumetric constraint, Lagrange multipliers will reside in the interior of the domain. We refer to this as a realisation of Neumann boundary conditions.
} 
system is then expanded to the form

$$
\left[\begin{array}{ccc}
\boldsymbol{K} & -\boldsymbol{A}^{T} & -\boldsymbol{B}^{T} \\
-\boldsymbol{A} & \mathbf{0} & \mathbf{0} \\
-\boldsymbol{B} & \mathbf{0} & \mathbf{0}
\end{array}\right]\left[\begin{array}{c}
\boldsymbol{u} \\
\hat{\boldsymbol{\tau}} \\
\hat{\boldsymbol{\sigma}}_{\mathrm{s}}
\end{array}\right]=\left[\begin{array}{c}
\mathbf{0} \\
-\overline{\boldsymbol{s}} \\
-\overline{\boldsymbol{g}}
\end{array}\right]
$$

from which the displacements and Lagrange multipliers can be solved for iteratively. Matrices $\boldsymbol{A}$ and $\boldsymbol{B}$ are the discretised versions of the homogenisation functions $\overline{\boldsymbol{s}}_{\square}(\boldsymbol{u})$ and $\overline{\boldsymbol{g}}_{\square}(\boldsymbol{u})$.

\section{APPLICATION EXAMPLE}

To illustrate the influence of the chosen definition of macroscopic reinforcement slip, a series of rebar pull-out tests was simulated. In the test, three $\phi 20$ rebars with lengths $0.2 \mathrm{~m}, 0.4 \mathrm{~m}$, and $0.6 \mathrm{~m}$, respectively, were pulled out of a square concrete RVE, with the side length equal to the length of the rebar. The pull-out was carried out by prescribing a given macroscopic slip via chosen boundary condition (boundary or volumetric definition of effective slip). In this case, the macroscopic reinforcement slip was imposed in steps of $\Delta \bar{s}=5 \times 10^{-5} \mathrm{~m}$. The bond-slip input for the rebars is schematically shown in Figure 1, and the parameters of the interface are summarised in Table 1, The novel boundary condition types were implemented in the open source $\mathrm{C}++$ code OOFEM (wWw. oofem.org) [18].

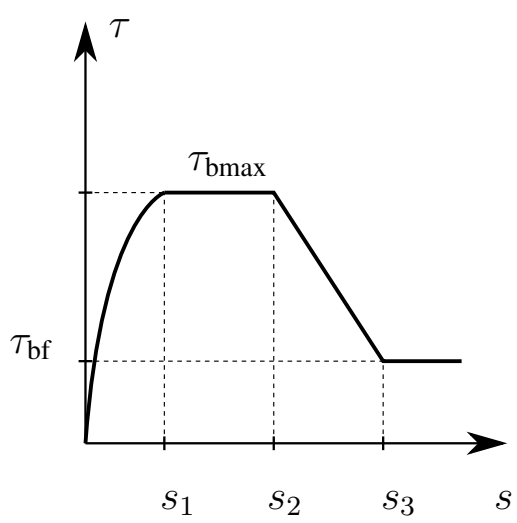

Figure 1: Bond-slip relation for good bond conditions [17].
Table 1: Interface parameters.

\begin{tabular}{cc}
\hline$s_{1}$ & $1.0 \mathrm{~mm}$ \\
\hline$s_{2}$ & $2.0 \mathrm{~mm}$ \\
\hline$s_{3}$ & $6.5 \mathrm{~mm}$ \\
\hline$\tau_{\text {bmax }}$ & $15.4 \mathrm{MPa}$ \\
\hline$\tau_{\text {bf }}$ & $6.2 \mathrm{MPa}$ \\
\hline
\end{tabular}

From the simulations, it was of interest to obtain the effective bond stress-slip relation for the different rebars. Hence, the average bond stress, $\bar{t}_{\Gamma}$ was calculated at each load step and is plotted against the macroscopic slip in Figure 2 for the boundary definition of slip. As can be seen in the figure, the response is highly dependent on the length of the rebar. The bond-slip input was recovered for the shortest rebars, while the effective response deviated much more from it for the longer bars. This is caused by the fact that the macroscopic slip is only defined at the boundary (ends) of the reinforcement bars. In order to illustrate this phenomenon, subscale results were consulted, and the local reinforcement slip was plotted along the rebars in Figure 3 ,

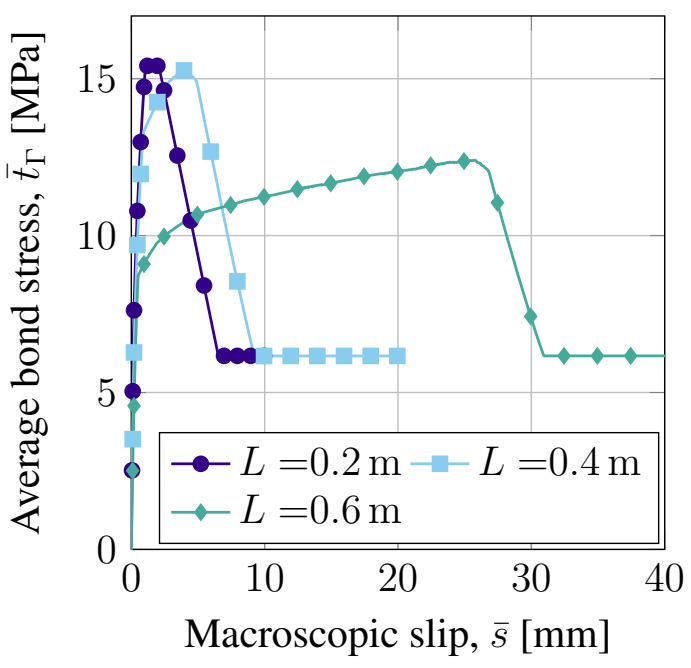

Figure 2: Average bond stress-macroscopic slip relations for reinforcement bars of different lengths using the boundary definition of macroscopic slip. 


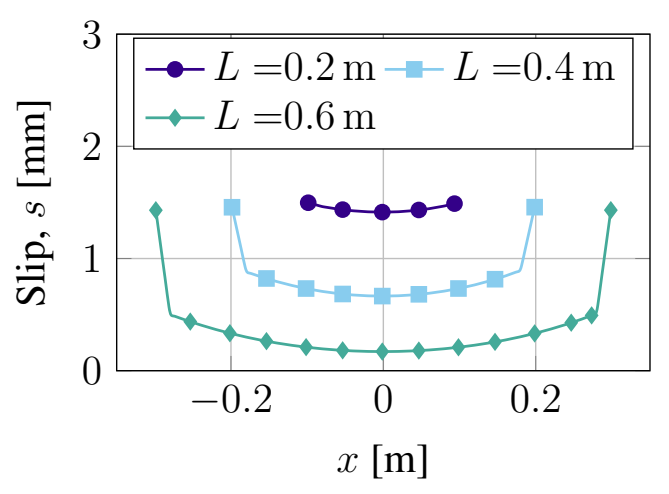

Figure 3: Reinforcement slip variation along the rebars of different lengths at the macroscopic slip $1.5 \mathrm{~mm}$ obtained with the boundary definition of macroscopic slip.

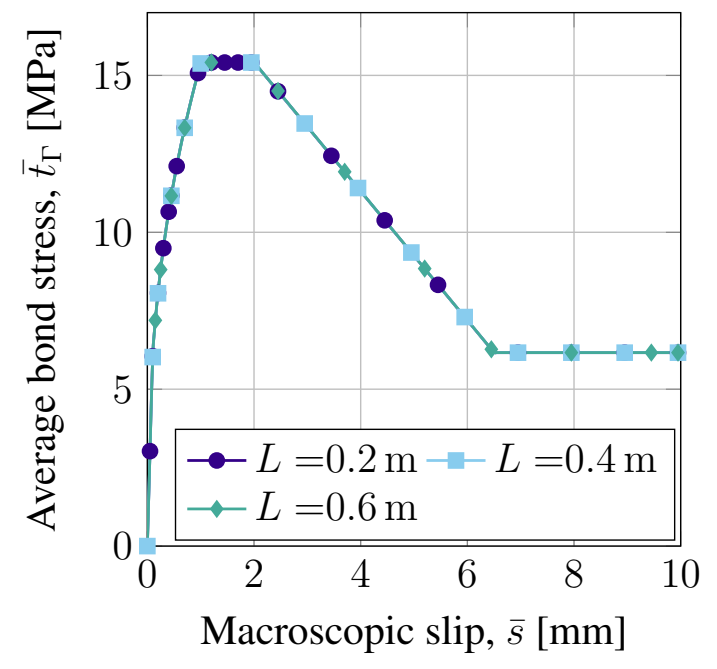

Figure 4: Average bond stress-macroscopic slip relations for reinforcement bars of different lengths using the volumetric definition of macroscopic slip.

Looking at the slip profiles in Figure 5, it can be seen that the local slip is uniformly distributed along the rebars, reaching the macroscopic value not only at the ends (boundary), but also in the interior of the reinforcement bars. Note that the local slip does not need to be constant in the RVE. This only holds true in this specific example. The results signify that this volumetric definition of the macroscopic slip is objective across the scales, i.e., the physical interpretation of the effective slip variable is not dependent on the size of the RVE.

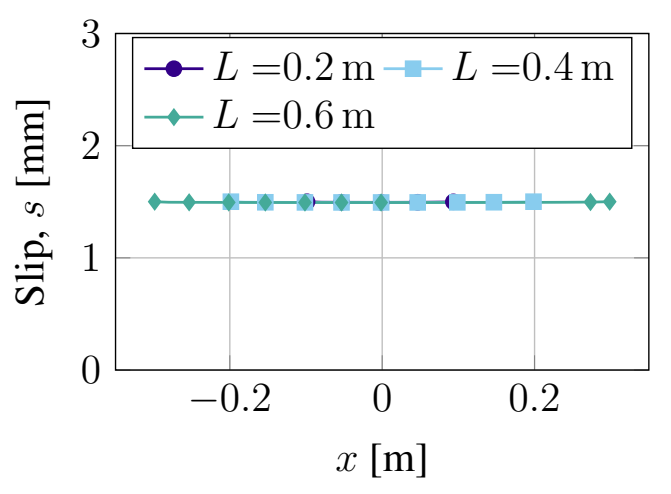

Figure 5: Reinforcement slip variation along the rebars of different lengths at the macroscopic slip $1.5 \mathrm{~mm}$ obtained with the volumetric definition of macroscopic slip. 


\section{CONCLUSIONS}

In this contribution, the issue of micro-tomacro transition of reinforcement slip in twoscale modelling of reinforced concrete is furhter studied. Two different definitions of the effective reinforcement slip are considered, namely a boundary and a volumetric one. By the former definition, the macroscopic variable is prescribed only at the boundary of the Representative Volume Element (RVE), and can be implemented in a straightforward form of Dirichlet boundary conditions. The latter definitions requires that the average local slip (as well as its gradient) along the reinforcement bars is equal to the macroscopic one. These constraints are applied on the RVE in form of Lagrange multipliers, which corresponds to Neumann boundary conditions, and the subscale problem can then be solved iteratively.

In a series of reinforcement pull-out tests it is demonstrated that the boundary definition of slip gives rise to highly RVE-size dependent response, which is undesirable from the modelling point of view. The volumetric definition of effective reinforcement slip acted as a remedy to this pathological RVE-size dependence of the effective bond-slip response. For future work, the novel volumetric micro-to-macro transition should be studied on structures globally, i.e., the influence of the new type of boundary conditions on the structural response in the two-scale model is of interest.

\section{REFERENCES}

[1] Nilenius, F., Larsson, F., Lundgren, K., Runesson, K. 2013. Macroscopic diffusivity in concrete determined by computational homogenization, International Journal for Numerical and Analytical Methods in Geomechanics 37(11), pp. 1535-1551.

[2] Nilenius, F., Larsson, F., Lundgren, K., Runesson, K. 2014. Computational homogenization of diffusion in three-phase mesoscale concrete, Computational Mechanics 54(2), pp. 461-472.
[3] Nilenius, F., Larsson, F., Lundgren, K., Runesson, K. 2015. Mesoscale modelling of crack-induced diffusivity in concrete, Computational Mechanics 55(2), pp. 359370.

[4] Fib, 2000. Bond of reinforcement in concrete: state-of-the-art report.

[5] Daoud, A., Maurel, O., Laborderie, C. 2013. 2D mesoscopic modelling of barconcrete bond, Engineering Structures 49; pp. 696-706.

[6] Unger, J., Eckardt, S. 2011. Multiscale Modeling of Concrete, Archives of Computational Methods in Engineering 18(3), pp. 341-393.

[7] Matouš, K., Geers, M.G.D., Kouznetsova, V., Gillman, A. 2017. A review of predictive nonlinear theories for multiscale modeling of heterogeneous materials, Journal of Computational Physics 330, pp. 192220.

[8] Feyel, F., Chaboche, J-L. 2000. FE2 multiscale approach for modelling the elastoviscoplastic behaviour of long fibre $\mathrm{SiC} / \mathrm{Ti}$ composite materials, Computer Methods in Applied Mechanics and Engineering 183(3), pp. 309-330.

[9] Feyel, F. 2003. A multilevel finite element method (FE2) to describe the response of highly non-linear structures using generalized continua, Computer Methods in Applied Mechanics and Engineering 192(28), pp. 3233-3244.

[10] Lackner, R., Mang, H. 2003. Scale Transition in Steel-Concrete Interaction. I: Model, Journal of Engineering Mechanics 129(4), pp. 393-402.

[11] Lackner, R., Mang, H. 2003. Scale Transition in Steel-Concrete Interaction. II: Applications, Journal of Engineering Mechanics 129(4), pp. 403-413. 
[12] Sciegaj, A., Larsson, F., Lundgren, K., Nilenius, F., and Runesson, K. 2019. Two-scale finite element modelling of reinforced concrete structures: Effective response and subscale fracture development, International Journal for Numerical Methods in Engineering 114(10); pp. 1074-1102.

[13] Sciegaj, A., Larsson, F., Lundgren, K., Nilenius, F., and Runesson, K. 2019. A multiscale model for reinforced concrete with macroscopic variation of reinforcement slip, Computational Mechanics 63(2); pp. 139-158.

[14] Larsson, F., Runesson, K., Su, F. 2010. Variationally consistent computational homogenization of transient heat flow. International Journal for Numerical Methods in Engineering 81(13); pp. 1659-1686.
[15] Geers, M., Kouznetsova, V., Brekelmans, W. 2003. MultiScale First-Order and Second-Order Computational Homogenization of Microstructures towards Continua, International Journal for Multiscale Computational Engineering 1(4), pp. 371-386.

[16] Karamnejad, A., Nguyen, V.P., Sluys, L.J. 2013. A multi-scale rate dependent crack model for quasi-brittle heterogeneous materials, Engineering Fracture Mechanics 104, pp. 96-113

[17] Model Code for Concrete Structures 2010. Wiley-VCH Verlag GmbH \& Co. KGaA, 2013

[18] Patzák, B., OOFEM - an object-oriented simulation tool for advanced modeling of materials and structures, Acta Polytechnica 52(6), pp. 59-66. 